University of Nebraska - Lincoln

DigitalCommons@University of Nebraska - Lincoln

September 2007

\title{
New Developments in Selenium Biochemistry: Selenocysteine Biosynthesis in Eukaryotes and Archaea
}

\author{
Xue-Ming Xu \\ National Institutes of Health, Bethesda, MD \\ Bradley A. Carlson \\ National Institutes of Health, Bethesda, MD \\ Yan Zhang \\ University of Nebraska-Lincoln, yzhang3@unl.edu \\ Heiko Mix \\ University of Nebraska-Lincoln \\ Gregory V. Kryukov \\ University of Nebraska-Lincoln \\ See next page for additional authors
}

Follow this and additional works at: https://digitalcommons.unl.edu/biochemgladyshev

Part of the Biochemistry, Biophysics, and Structural Biology Commons

Xu, Xue-Ming; Carlson, Bradley A.; Zhang, Yan; Mix, Heiko; Kryukov, Gregory V.; Glass, Richard S.; Berry, Marla J.; Gladyshev, Vadim N.; and Hatfield, Dolph L., "New Developments in Selenium Biochemistry: Selenocysteine Biosynthesis in Eukaryotes and Archaea" (2007). Vadim Gladyshev Publications. 44. https://digitalcommons.unl.edu/biochemgladyshev/44

This Article is brought to you for free and open access by the Biochemistry, Department of at DigitalCommons@University of Nebraska - Lincoln. It has been accepted for inclusion in Vadim Gladyshev Publications by an authorized administrator of DigitalCommons@University of Nebraska - Lincoln. 


\section{Authors}

Xue-Ming Xu, Bradley A. Carlson, Yan Zhang, Heiko Mix, Gregory V. Kryukov, Richard S. Glass, Marla J.

Berry, Vadim N. Gladyshev, and Dolph L. Hatfield 


\title{
New Developments in Selenium Biochemistry: Selenocysteine Biosynthesis in Eukaryotes and Archaea
}

\author{
Xue-Ming Xu • Bradley A. Carlson • Yan Zhang • \\ Heiko Mix • Gregory V. Kryukov • Richard S. Glass • \\ Marla J. Berry • Vadim N. Gladyshev • \\ Dolph L. Hatfield
}

Published online: 1 August 2007

\begin{abstract}
We used comparative genomics and experimental analyses to show that (1) eukaryotes and archaea, which possess the selenocysteine (Sec) protein insertion machinery contain an enzyme, $O$-phosphoseryl-transfer RNA (tRNA) ${ }^{[\mathrm{Ser}] \mathrm{Sec}}$ kinase (designated PSTK), which phosphorylates seryl-tRNA ${ }^{[\mathrm{Ser}] \mathrm{Sec}}$ to form $O$-phosphoseryl-tRNA ${ }^{[\mathrm{Ser}] \mathrm{Sec}}$ and (2) the Sec synthase (SecS) in mammals is a pyridoxal phosphate-containing protein previously described as the soluble liver antigen (SLA). SecS uses the product of PSTK, $O$ phosphoseryl-tRNA ${ }^{[\mathrm{Ser}] \mathrm{Sec}}$, and selenophosphate as substrates to generate selenocysteyltRNA $^{[\mathrm{Ser}] \mathrm{Sec}}$. Sec could be synthesized on $\mathrm{tRNA}^{[\mathrm{Ser}] \mathrm{Sec}}$ from selenide, adenosine triphosphate (ATP), and serine using tRNA ${ }^{[\mathrm{Ser}] \mathrm{Sec}}$, seryl-tRNA synthetase, PSTK, selenophosphate synthetase, and SecS. The enzyme that synthesizes monoselenophosphate is a previously identified selenoprotein, selenophosphate synthetase 2 (SPS2), whereas the previously identified mammalian selenophosphate synthetase 1 did not serve this function. Monoselenophosphate also served directly in the reaction replacing ATP, selenide, and SPS2, demonstrating that this compound was the active selenium donor. Conservation of the overall pathway of Sec biosynthesis suggests that this pathway is also active in other eukaryotes and archaea that contain selenoproteins.
\end{abstract}

X.-M. Xu and B. A. Carlson contributed equally to the studies described herein.

X.-M. Xu • B. A. Carlson • D. L. Hatfield $(\bowtie)$

Molecular Biology of Selenium Section, Laboratory of Cancer Prevention, Center for Cancer Research, National Institutes of Health, Bethesda, MD 20892, USA

e-mail: hatfield@dc37a.nci.nih.gov

Y. Zhang $\cdot$ H. Mix $\cdot$ G. V. Kryukov $\cdot$ V. N. Gladyshev

Department of Biochemistry, University of Nebraska, Lincoln, NE 68588, USA

R. S. Glass

Department of Chemistry, The University of Arizona, Tucson, AZ 85721, USA

M. J. Berry

Department of Cell and Molecular Biology, University of Hawaii at Manoa, Honolulu, HI 96813, USA 
Keywords Selenocysteine - Selenocysteine biosynthesis · Selenocysteine synthase · Selenophosphate synthetase $\cdot$ Selenoproteins

\section{Introduction}

The selenium field has grown at a rapid pace over the last 15 to 20 years, and many new developments have occurred recently in the biochemistry and genetic areas of selenium research. Several thorough reviews have covered these topics [1-4]. Herein, we have focused on recent developments involving the biosynthesis of selenocysteine ( $\mathrm{Sec})$ in eukaryotes and archaea that have not been reviewed elsewhere.

$\mathrm{Sec}$ is the $21 \mathrm{st}$ amino acid in the genetic code. It is cotranslationally inserted into protein in response to the codon, UGA [1-4]. The biosynthesis of Sec occurs on its transfer RNA (tRNA), and the pathway begins with the initial aminoacylation of Sec tRNA with serine [5, 6]. Thus, the backbone for Sec is serine, and the identity elements in Sec tRNA for its aminoacylation correspond to those in seryl-tRNA synthetase [7, 8]. Sec tRNA has therefore been appropriately designated Sec tRNA ${ }^{[\mathrm{Ser}] \mathrm{Sec}}$ [9]. Although the biosynthetic pathway for Sec was established in eubacteria more than 15 years ago [10-12], only recently was the complete pathway for the biosynthesis of this amino acid elucidated in eukaryotes [13]. In Escherichia coli, the enzyme that synthesizes Sec, Sec synthase (SelA), was identified in the early 1990s and is a pyridoxal phosphate (PLP)-dependent protein $[10-12]$. SelA acts on the serine moiety attached to Sec tRNA ${ }^{[\operatorname{Ser}] \operatorname{Sec}}$ by initially removing the hydroxyl group from serine to generate aminoacrylyl as an intermediate. Aminoacrylyl then accepts activated selenium to form selenocysteyl-tRNA ${ }^{[\mathrm{Ser}] \mathrm{Sec}}$. The activated selenium in bacteria is monoselenophosphate, and it is synthesized from selenide and adenosine triphosphate (ATP) by E. coli selenophosphate synthetase (SelD) [14].

Several factors were previously characterized in higher vertebrates that were found to have a role in Sec biosynthesis. For example, a kinase that phosphorylated a minor seryltRNA to form $O$-phosphoseryl-tRNA was reported in rooster liver as early as 1970 [15]. This same year a minor seryl-tRNA from mammalian and chicken liver was found to decode specifically the termination codon, UGA [16], which was subsequently shown to be the same tRNA that formed phosphoseryl-tRNA [17]. This tRNA was subsequently identified as Sec tRNA ${ }^{[\mathrm{Ser}] \mathrm{Sec}}[6]$. The kinase, designated $O$-phosphoseryl-tRNA ${ }^{[\mathrm{Ser}] \mathrm{Sec}}$ kinase (PSTK), was recently isolated, characterized, and found to specifically phosphorylate the seryl moiety on seryl-tRNA ${ }^{[\mathrm{Ser}] \mathrm{Sec}}[18]$. The resulting $O$-phosphoseryltRNA $^{[\mathrm{Ser}] \mathrm{Sec}}$ product had been proposed as an intermediate in the biosynthesis of $\mathrm{Sec}[3$, 18]. In addition, because no sequences with homology to SelA could be detected in eukaryotes, there was some question as to the identity of the Sec synthase (SecS) in mammals. The soluble liver antigen (SLA), which was initially identified as a 48-kDa protein bound to Sec tRNA ${ }^{[\mathrm{Ser}] \mathrm{Sec}}$ and was targeted by antibodies in patients with an autoimmune chronic hepatitis [19], was reported to occur as a separate family within a larger superfamily of diverse PLP-dependent transferases [20] and proposed as a possible $\mathrm{SecS}$ in mammals [3, 20-22]. SLA was also found to exist in a protein complex with other factors involved in Sec biosynthesis and/or its insertion into protein providing further evidence that this protein is involved in selenium metabolism [22, 23]. As described herein, SLA is indeed SecS in mammals [13]. In addition, two genes, sps 1 and sps2, were found that had homology to SelD and the products of both genes were initially proposed to serve as selenophosphate synthetase (SPS) in mammals [24-27]. Interestingly, sps 2 encodes a selenoprotein, SPS2, which can serve, provided it is responsible for making the active 
selenium donor for the biosynthesis of Sec, as an autoregulator of selenoprotein synthesis $[24,26]$. The Sec moiety in SPS2 was mutated to Cys, and the mutant has low enzyme activity $[24,26,28]$, but is capable of complementing SelD $^{-}$E. coli cells transfected with the mammalian mutant form [28]. Additional studies involving the complementation of SelD $^{-}$E. coli cells with either SPS2 or SPS1 suggested that SPS2 is involved in the synthesis of selenophosphate, whereas SPS1 has a role in recycling Sec via a selenium salvage pathway [29].

We used a computational, comparative genomics approach to identify a single kinase with homologies common only to archaea and eukaryotes that utilize the Sec protein insertion machinery [18]. We also used a similar comparative genomics approach to identify SLA as the likely SecS [13]. These two studies demonstrate the potential of scanning even very distantly related genomes for new metabolic enzymes and the value of interdisciplinary research.

Each of the components discussed above, including tRNA ${ }^{[\mathrm{Ser}] \mathrm{Sec}}$, seryl-tRNA ${ }^{[\mathrm{Ser}] \mathrm{Sec}}$, $O$-phosphoseryl-tRNA ${ }^{[\mathrm{Ser}] \mathrm{Sec}}$, seryl-tRNA ${ }^{[\mathrm{Ser}] \mathrm{Sec}}$ synthetase, PSTK, SLA, SPS2, and selenophosphate, finally were used to establish the biosynthetic pathway of the 21 st amino acid in the genetic code in eukaryotes [13]. The fact that both SLA and PSTK sequences are found in eukaryotes and archaea that utilize the Sec protein insertion machinery, but not in those organisms that do not, provide strong evidence that this pathway is used by all eukaryotes and archaea that synthesize Sec-containing proteins $([13,18]$ and also see [30]).

\section{Discussion}

Identifying Sec Kinase

We initially hypothesized that the pathway for synthesizing Sec was the same in those eukaryotes and archaea-making selenoproteins and that $O$-phosphoseryl-tRNA was a common intermediate [18]. Therefore, we compared the genomes of the two archaea that encode the Sec protein insertion machinery, Methanopyrus kandleri and Methanococccus jannaschii, to the 12 archaea whose genomes have been sequenced and do not encode the Sec insertion machinery for kinase-like genes using the BLASTN program [18]. There were two candidates found in the two archaea using Sec insertion machinery and not in the 12 other archaea. Interestingly, only one of these two protein genes, a predicted nucleotide kinase (gi: 20095115), had homologous sequences in eukaryotes encoding the Sec insertion machinery, but not in those eukaryotes whose genomes had been sequenced and lacked the Sec insertion machinery. The corresponding putative kinase gene ( $p s t k)$ from mice was cloned into an expression vector, expressed, and the product purified and characterized [18]. The pstk coding sequence is 1,080 nucleotides that encodes a 359 amino acid protein. The resulting protein generated from the cloned expression vector used the serylated forms of $\mathrm{mcm}^{5} \mathrm{U}$ and $\mathrm{mcm}^{5} \mathrm{Um}$ as substrates to yield $O$-phosphoseryl-tRNA ${ }^{[\mathrm{Ser}] \mathrm{Sec}}$. In addition to these two serylated isoforms, PSTK requires ATP and $\mathrm{Mg}^{++}$, and the reaction may be summarized as follows:

\section{Seryl-tRNA ${ }^{[\mathrm{Ser}] \mathrm{Sec}}+\mathrm{ATP} \stackrel{\left(\mathrm{PSTK}+\mathrm{Mg}^{++)}\right.}{\leftarrow} O$-phosphoseryl-tRNA ${ }^{[\mathrm{Ser}] \mathrm{Sec}}+\mathrm{ADP}$}

Even after the identification and characterization of PSTK [18], its function was uncertain, as this kinase had been proposed to provide either a storage form of Sec 
$\mathrm{tRNA}^{[\mathrm{Ser}] \mathrm{Sec}}[31]$ or the intermediate in Sec biosynthesis $[3,18]$. This issue was not resolved until the entire biosynthetic pathway of $\mathrm{Sec}$ was determined as described below.

\section{Identifying SecS}

The genomes of eukaryotes and archaea that had been completely sequenced were analyzed for the occurrence of selenoproteins, and comparative genomics studies were carried out to identify genes that co-occur with selenoproteins in these organisms [13]. Each search resulted in known components of the Sec insertion machinery and an additional protein that might be SecS in mammals. This additional protein had previously been detected in patients with autoimmune chronic hepatitis as an autoimmune factor that co-precipitated $\mathrm{RRNA}^{[\mathrm{Ser}] \mathrm{Sec}}$ in cell extracts of these individuals and was known as the SLA [19]. SLA, which is a PLPdependent transferase [20], also bound other protein components involved with Sec metabolism [22, 23]. We found that SLA occurred in all eukaryotes and archaea-encoding selenoproteins, but not in those organisms examined that lacked selenoproteins. These and other observations strongly suggested that SLA was the missing SecS in eukaryotes and archaea and that SelA from eubacteria and this potential SecS belonged to totally different families of PLP-containing proteins suggesting that their similar functions arose by convergent evolution [13].

After identifying a potential SecS candidate by comparative genomics analysis as described above, we examined its function experimentally. The putative mouse $\operatorname{Sec} S(\mathrm{mSecS})$ gene was cloned and expressed, and the binding of $O$-phosphoseryl-tRNA ${ }^{[\mathrm{Ser}] \mathrm{Sec}}$, seryltRNA $^{[\mathrm{Ser}] \mathrm{Sec}}$ and tRNA ${ }^{[\mathrm{Ser}] \mathrm{Sec}}$, and seryl-tRNA ${ }^{\mathrm{Ser}}$ and $\mathrm{tRNA}^{\mathrm{Ser}}$ as controls, to the expressed product were examined. The efficiency of binding was $O$-phosphoseryl-tRNA ${ }^{[\mathrm{Ser}] \mathrm{Sec}}>$ tRNA $^{[\mathrm{Ser}] \mathrm{Sec}}>$ seryl-tRNA $^{[\mathrm{Ser}] \mathrm{Sec}}$, whereas seryl-tRNA ${ }^{\mathrm{Ser}}$ and $\mathrm{tRNA}^{\mathrm{Ser}}$ did not bind at all. These results suggested that $O$-phosphoseryl-tRNA ${ }^{[\mathrm{Ser}] \mathrm{Sec}}$ is a substrate for mSecS. We next tested whether mSecS would remove the phosphate from the $O$-phosphoseryl moiety and found that it was rapidly dephosphorylated. The dephosphorylated product is likely not seryl-tRNA ${ }^{[\mathrm{Ser}] \mathrm{Sec}}$, as the product bound efficiently to mSecS, but seryl-tRNA ${ }^{[\mathrm{Ser}] \mathrm{Sec}}$ did not. Although these experiments suggested that $\mathrm{mSecS}$ was the mammalian SecS, it was essential to determine that the dephosphorylated product would accept the active selenium donor to form Sec before it could be concluded that mouse SLA was indeed the true Sec synthase in mammals. We therefore turned our attention to examining the functions of the two SPS, SPS1 and SPS2, to determine if either, or both, could produce the active selenium donor.

\section{Identifying a Functional SPS in Mammals}

To identify the active selenium donor, mouse sps 1 (msps1) and sps2 (msps2) were individually cloned into an expression vector, and the Sec codon in msps 2 converted to a Cys codon (msps2-Cys) [13]. Furthermore, because Caenorhabditis elegans sps2 (csps2) naturally encodes Cys at the active site in place of Sec found in mammals, csps 2 was also cloned. We expressed msps 1, msps2-Cys, csps2, and E. coli selD, purified the products, and assessed their abilities to synthesize monoselenophosphate ( $\mathrm{SeP}$ ) because, as noted in "Introduction," SeP is synthesized by SelD and had been identified as the active selenium donor in E. coli [10-12]. Each of these proteins was incubated separately with ATP and selenite under anaerobic conditions and a ${ }^{31} \mathrm{P}$ NMR spectrophotometric analysis of the products carried out. The mSPS2-Cys, cSPS2, and SelD reactions produced a signal at $+23.2 \mathrm{ppm}$ that corresponded to SeP, but interestingly, mSPS1 did not. These data provide strong evidence that the eukaryotic SPS2s synthesize SeP, as does SelD, but SPS1 does not. 
Sec Biosynthesis (In Vitro)

As previous studies analyzing Sec biosynthesis had not determined whether SeP could serve directly as the selenium donor [10-12], we chemically synthesized this compound and used it in in vitro reactions. Reactions containing $\mathrm{SeP}$ and $O$-phosphoseryl-tRNA ${ }^{[\mathrm{Ser}] \mathrm{Sec}}$ incubated in the presence of $\mathrm{mSecS}$ did indeed produce Sec. Control assays containing seryl-tRNA ${ }^{[\mathrm{Ser}] \mathrm{Sec}}$ in place of $O$-phosphoseryl-tRNA ${ }^{[\mathrm{Ser}] \mathrm{Sec}}$, without $\mathrm{SeP}$ or containing another protein in place of $\mathrm{mSecS}$, did not form Sec. These studies unequivocally demonstrated that $\mathrm{SeP}$ is the active selenium donor in Sec biosynthesis in which SLA is the missing SecS and $O$-phosphoseryl-tRNA ${ }^{[\mathrm{Ser}] \mathrm{Sec}}$ is a true intermediate in the Sec biosynthetic pathway [13].

Although, as noted above, SPS2 was capable of producing SeP in reactions with ATP and selenide, whereas SPS1 was not, it was important to demonstrate whether Sec could be produced on its tRNA in reactions with these components and $O$-phosphoseryl-tRNA ${ }^{[\mathrm{Ser}] \mathrm{Sec}}$ and SecS. Incubation of mSecS, $O$-phosphoseryl-tRNA ${ }^{[\mathrm{Ser}] \mathrm{Sec}}, \mathrm{mSPS} 2$, selenide, and ATP produced Sec. However, replacement of mSecS with mSPS1 or $O$-phosphoseryl-tRNA ${ }^{[\mathrm{Ser}] \mathrm{Sec}}$ with seryl-tRNA ${ }^{[\mathrm{Ser}] \mathrm{Sec}}$ did not generate Sec. These studies demonstrate unequivocally that SPS2 synthesizes the active selenium donor for Sec synthesis and that SPS1 likely has another role in selenium metabolism. It is of interest to note that we also observed that SelA could use either seryl-tRNA ${ }^{[\mathrm{Ser}] \mathrm{Sec}}$ or phosphoseryl-tRNA ${ }^{[\mathrm{Ser}] \mathrm{Sec}}$ as a substrate to produce $\mathrm{Sec}$ in the presence of either SeP.

The intermediate generated by SelA action on seryl-tRNA ${ }^{[\mathrm{Ser}] \mathrm{Sec}}$ has been identified as dehydroalanyl-tRNA ${ }^{[\mathrm{Ser}] \mathrm{Sec}}[10-12]$. Although we did not identify the intermediate synthesized by the dephosphorylation of $O$-phosphoseryl-tRNA ${ }^{[\mathrm{Ser}] \mathrm{Sec}}$ by $\mathrm{mSecS}$, the available evidence suggested that it also was dehydroalanyl-tRNA ${ }^{[\mathrm{Ser}] \mathrm{Sec}}[13]$.

\section{Sec Biosynthesis (In Vivo)}

A previous study had established SPS1 as a mammalian SPS [27], whereas another report had suggested that SPS1 utilized free Sec for the biosynthesis of selenoproteins [29]. Our in vitro studies described above, however, demonstrated that SPS2 is responsible for synthesizing SeP, whereas SPS1 likely has another role in selenium metabolism. To resolve these discrepancies between the earlier work [27, 29] and our more recent work [13] and to further elucidate the roles of SPS1 and SPS2 in vivo, we used RNAi technology to

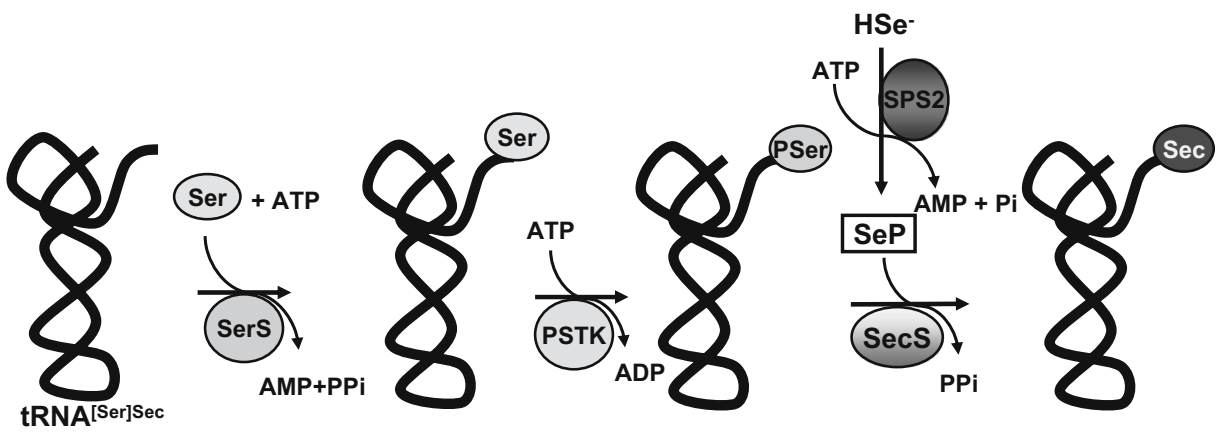

Fig. 1 The Sec biosynthetic pathway in eukaryotes. Abbreviations are defined in the text with the exception of SerS, which is seryl-tRNA synthetase 
knockdown SPS1 and SPS2 intracellularly and examine the effect(s) of their targeted removal on selenoprotein synthesis [32]. Knockdown of SPS2 in NIH3T3 cells obliterated selenoprotein synthesis, whereas knockdown of SPS1 had no affect. We then used the knockdown cells to transfect them with SPS2 or SPS1 and found that SPS2 or SelD complemented the function of the removed SPS2, but SPS1 did not. These in vivo studies demonstrated that SPS2, which synthesizes SeP [13] is essential for selenoprotein synthesis and that SPS1 likely has a more specialized function in selenium metabolism [32].

\section{The Sec Biosynthetic Pathway}

The entire biosynthetic pathway of Sec in eukaryotes is shown in Fig. 1. tRNA $^{[\mathrm{Ser}] \mathrm{Sec}}$ is initially aminoacylated with serine by seryl-tRNA synthetase [5, 6]. The seryl moiety is then phosphorylated by PSTK to form $O$-phosphoseryl-tRNA ${ }^{[\mathrm{Ser}] \mathrm{Sec}}[18]$. $O$-phosphoseryltRNA $^{[\mathrm{Ser}] \mathrm{Sec}}$ is the substrate for SecS, which in turn hydrolyzes the phosphate group to form the acceptor molecule for $\mathrm{SeP}$, and the latter reaction, which is also carried out by SecS, yields Sec. SPS2 generates the active selenium donor, SeP, from selenide and ATP [13].

The kinase, PSTK, is not found in eubacteria, but interestingly, SelA can use $O$ phosphoseryl-tRNA ${ }^{[\mathrm{Ser}] \mathrm{Sec}}$ as a substrate. The major difference in the biosynthesis of Sec in eubacteria and the pathway described herein in eukaryotes is the extra step involving $O$ phosphoseryl-tRNA ${ }^{[\mathrm{Ser}] \mathrm{Sec}}$ synthesis, which is a substrate for SecS [13, 30], whereas seryltRNA is the substrate for SelA in E. coli.

\section{Concluding Remarks}

The biosynthesis of Sec, (1) from the initial step involving the aminoacylation of tRNA ${ }^{[\mathrm{Ser}] \mathrm{Sec}}$ with serine by seryl-tRNA synthetase (2) through its phosphorylation by PSTK to form $O$ phosphoseryl-tRNA ${ }^{[\mathrm{Ser}] \mathrm{Sec}}$, (3) subsequent dephosphorylation of $O$-phosphoseryltRNA $^{[\mathrm{Ser}] \mathrm{Sec}}$ to yield the acceptor molecule for SeP, (4) that in turn forms Sec wherein the last two steps are carried out by SecS, has been deciphered in mammals (Fig. 1). We also determined that SeP is synthesized by SPS2 and not by SPS1. These findings demonstrate how selenium, an essential micronutrient in the diet of humans and other mammals, becomes a component of $\mathrm{Sec}$ for its incorporation into protein in eukaryotes and archaea. Selenoproteins are thought to be responsible for most of the health benefits of selenium, which include preventing cancer and heart disease, delaying the aging process and the onset of AIDS in HIV positive patients, and roles in development and immune function [4].

Acknowledgements This research was supported by the Intramural Research Program of the National Institutes of Health, National Cancer Institute, and Center for Cancer Research, by grants GM061603, GM065204, and CA080946 to VNG, grants DK47320 and DK52963 to MJB, and grant CA-41108 (NIH Colon Cancer Prevention Program Project) to RSG.

\section{References}

1. Birringer M, Pilawa S, Flohe L (2002) Trends in selenium biochemistry. Nat Prod Rep 19:693-718

2. Driscoll DM, Copeland PR (2003) Mechanism and regulation of selenoprotein synthesis. Annu Rev Nutr 23:17-40 
3. Hatfield DL, Carlson BA, Xu XM, Mix H, Gladyshev VN (2006) Selenocysteine incorporation machinery and the role of selenoproteins in development and health. Prog Nucleic Acid Res Mol Biol 81:97-142

4. Hatfield DL, Berry MJ, Gladyshev VN, Eds (2006) Selenium: its molecular biology and role in human health. Springer

5. Leinfelder W, Stadtman TC, Bock A (1989) Occurrence in vivo of selenocysteyl-tRNA(SERUCA) in Escherichia coli. Effect of sel mutations. J Biol Chem 264:9720-9723

6. Lee BJ, Worland PJ, Davis JN, Stadtman TC, Hatfield DL (1989) Identification of a selenocysteyl-tRNA (Ser) in mammalian cells that recognizes the nonsense codon, UGA. J Biol Chem 264:9724-9727

7. Wu XQ, Gross HJ (1993) The long extra arms of human tRNA([Ser]Sec) and tRNA(Ser) function as major identify elements for serylation in an orientation-dependent, but not sequence-specific manner. Nucleic Acids Res 21:5589-5594

8. Ohama T, Yang DC, Hatfield DL (1994) Selenocysteine tRNA and serine tRNA are aminoacylated by the same synthetase, but may manifest different identities with respect to the long extra arm. Arch Biochem Biophys 315:293-301

9. Hatfield DL, Choi IS, Ohama T, Jung JE, Diamond AM (1994) Selenocysteine tRNA(Ser)sec isoacceptors as central components in selenoprotein biosynthesis in eukaryotes. In: Burk RF (ed) Selenium in biology and human health. Springer, New York, NY, pp 25-44

10. Forchhammer K, Leinfelder W, Boesmiller K, Veprek B, Bock A (1991) Selenocysteine synthase from Escherichia coli. Nucleotide sequence of the gene (selA) and purification of the protein. J Biol Chem 266:6318-6323

11. Forchhammer K, Boesmiller K, Bock A (1991) The function of selenocysteine synthase and SELB in the synthesis and incorporation of selenocysteine. Biochimie 73:1481-1486

12. Forchhammer K, Bock A (1991) Selenocysteine synthase from Escherichia coli. Analysis of the reaction sequence. J Biol Chem 266:6324-6328

13. Xu XM, Carlson BA, Mix H et al (2007) Biosynthesis of selenocysteine on Its tRNA in Eukaryotes. PLoS Biol 5:96-105

14. Glass RS, Singh WP, Jung W, Veres Z, Scholz TD, Stadtman TC (1993) Monoselenophosphate: synthesis, characterization, and identity with the prokaryotic biological selenium donor, compound SePX. Biochemistry 32:12555-12559

15. Maenpaa PH, Bernfield MR (1970) A specific hepatic transfer RNA for phosphoserine. Proc Natl Acad Sci U S A 67:688-695

16. Hatfield DL, Portugal FH (1970) Seryl-tRNA in mammalian tissues: chromatographic differences in brain and liver and a specific response to the codon, UGA. Proc Natl Acad Sci U S A 67:1200-1206

17. Hatfield DL, Diamond AM, Dudock B (1982) Opal suppressor serine tRNAs from bovine liver form phosphoseryl-tRNA. Proc Natl Acad Sci U S A 79:6215-6219

18. Carlson BA, Xu XM, Kryukov GV et al (2004) Identification and characterization of phosphoseryltRNA[Ser]Sec kinase. Proc Natl Acad Sci U S A 101:12848-12853

19. Gelpi C, Sontheimer EJ, Rodriguez-Sanchez JL (1992) Autoantibodies against a serine tRNA-protein complex implicated in cotranslational selenocysteine insertion. Proc Natl Acad Sci U S A 89:9739-9743

20. Kernebeck T, Lohse AW, Grotzinger J (2001) A bioinformatical approach suggests the function of the autoimmune hepatitis target antigen soluble liver antigen/liver pancreas. Hepatology 34:230-233

21. Allmang C, Krol A (2006) Selenoprotein synthesis: UGA does not end the story. Biochimie 88:1561-1571

22. Small-Howard A, Morozova N, Stoytcheva Z et al (2006) Supramolecular complexes mediate selenocysteine incorporation in vivo. Mol Cell Biol 26:2337-2346

23. Xu XM, Mix H, Carlson BA et al (2005) Evidence for direct roles of two additional factors, SECp43 and soluble liver antigen, in the selenoprotein synthesis machinery. J Biol Chem 280:41568-41575

24. Guimaraes MJ, Peterson D, Vicari A et al (1996) Identification of a novel selD homolog from eukaryotes, bacteria, and archaea: is there an autoregulatory mechanism in selenocysteine metabolism? Proc Natl Acad Sci U S A 93:15086-15091

25. Kim IY, Stadtman TC (1995) Selenophosphate synthetase: detection in extracts of rat tissues by immunoblot assay and partial purification of the enzyme from the archaean Methanococcus vannielii. Proc Natl Acad Sci U S A 92:7710-7713

26. Kim IY, Guimaraes MJ, Zlotnik A, Bazan JF, Stadtman TC (1997) Fetal mouse selenophosphate synthetase 2 (SPS2): characterization of the cysteine mutant form overproduced in a baculovirus-insect cell system. Proc Natl Acad Sci U S A 94:418-421

27. Low SC, Harney JW, Berry MJ (1995) Cloning and functional characterization of human selenophosphate synthetase, an essential component of selenoprotein synthesis. J Biol Chem 270:21659-21664 
28. Kim TS, Yu MH, Chung YW et al (1999) Fetal mouse selenophosphate synthetase 2 (SPS2): biological activities of mutant forms in Escherichia coli. Mol Cells 9:422-428

29. Tamura T, Yamamoto S, Takahata M et al (2004) Selenophosphate synthetase genes from lung adenocarcinoma cells: Sps1 for recycling L-selenocysteine and Sps2 for selenite assimilation. Proc Natl Acad Sci U S A 101:16162-16167

30. Yuan J, Palioura S, Salazar JC et al (2006) RNA-dependent conversion of phosphoserine forms selenocysteine in eukaryotes and archaea. Proc Natl Acad Sci U S A 103:18923-18927

31. Amberg R, Mizutani T, Wu XQ, Gross HJ (1996) Selenocysteine synthesis in mammalia: an identity switch from tRNA(Ser) to tRNA(Sec). J Mol Biol 263:8-19

32. Xu X-M, Carlson BA, Irons R, Mix H, Zhong N, Gladyshev VN, Hatfield DL (2007) Selenophosphate synthetase 2 is essential for selenoprotein biosynthesis. Biochem J 404:115-120 